\title{
Article \\ Gut Microbiome in a Russian Cohort of Pre- and Post-Cholecystectomy Female Patients
}

\author{
Irina Grigor'eva ${ }^{1, *} \mathbb{\infty}$, Tatiana Romanova ${ }^{1}\left(\right.$, Natalia Naumova ${ }^{2, *}$, Tatiana Alikina ${ }^{2}$, Alexey Kuznetsov ${ }^{3}$ and \\ Marsel Kabilov ${ }^{2}$ (1) \\ 1 Research Institute of Internal and Preventive Medicine-Branch of the Institute of Cytology and Genetics, \\ Siberian Branch of Russian Academy of Sciences, Novosibirsk 630089, Russia; tarom_75@mail.ru \\ 2 Institute of Chemical Biology and Fundamental Medicine, Siberian Branch of the Russian Academy of \\ Sciences, Novosibirsk 630090, Russia; alikina@niboch.nsc.ru (T.A.); kabilov@niboch.nsc.ru (M.K.) \\ 3 Novosibirsk State Medical University, Novosibirsk 630091, Russia; 1xo2788353@mail.ru \\ * Correspondence: igrigorieva@ngs.ru (I.G.); naumova@niboch.nsc.ru (N.N.)
}

Citation: Grigor'eva, I.; Romanova,

T.; Naumova, N.; Alikina, T.;

Kuznetsov, A.; Kabilov, M. Gut

Microbiome in a Russian Cohort of Pre- and Post-Cholecystectomy Female Patients. J. Pers. Med. 2021, 11, 294. https://doi.org/10.3390/ jpm11040294

Academic Editor: Lucrezia Laterza

Received: 19 February 2021

Accepted: 8 April 2021

Published: 12 April 2021

Publisher's Note: MDPI stays neutral with regard to jurisdictional claims in published maps and institutional affiliations.

Copyright: (c) 2021 by the authors. Licensee MDPI, Basel, Switzerland. This article is an open access article distributed under the terms and conditions of the Creative Commons Attribution (CC BY) license (https:/ / creativecommons.org/licenses/by/ $4.0 /)$.

\begin{abstract}
The last decade saw extensive studies of the human gut microbiome and its relationship to specific diseases, including gallstone disease (GSD). The information about the gut microbiome in GSD-afflicted Russian patients is scarce, despite the increasing GSD incidence worldwide. Although the gut microbiota was described in some GSD cohorts, little is known regarding the gut microbiome before and after cholecystectomy (CCE). By using Illumina MiSeq sequencing of 16S rRNA gene amplicons, we inventoried the fecal bacteriobiome composition and structure in GSD-afflicted females, seeking to reveal associations with age, BMI and some blood biochemistry. Overall, 11 bacterial phyla were identified, containing 916 operational taxonomic units (OTUs). The fecal bacteriobiome was dominated by Firmicutes ( $66 \%$ relative abundance), followed by Bacteroidetes (19\%), Actinobacteria (8\%) and Proteobacteria (4\%) phyla. Most $(97 \%)$ of the OTUs were minor or rare species with $\leq 1 \%$ relative abundance. Prevotella and Enterocossus were linked to blood bilirubin. Some taxa had differential pre- and post-CCE abundance, despite the very short time (1-3 days) elapsed after CCE. The detailed description of the bacteriobiome in pre-CCE female patients suggests bacterial foci for further research to elucidate the gut microbiota and GSD relationship and has potentially important biological and medical implications regarding gut bacteria involvement in the increased GSD incidence rate in females.
\end{abstract}

Keywords: gallstone disease; $16 \mathrm{~S}$ rDNA gene diversity; gut microbiota; blood biochemical characteristics

\section{Introduction}

Gallstone disease (GSD) has been, for many years, a significant public health problem worldwide, and its prevalence rate is expected to increase due to the ongoing changes in lifestyle and dietary habits. Gallstones are highly prevalent in Russia, with 100,000-200,000 cholecystectomies performed annually [1,2].

By now, there is little doubt about the multifaceted relationship between GSD and the microbiota [3], as some intestinal bacteria can promote gallstone formation [4-6], particularly by modifying the bile acid profile [7]. However, laparoscopic cholecystectomy (CCE), albeit currently a radical gold standard treatment, is not a neutral event and may increase the risk of some serious disorders and diseases, including metabolic syndrome, cardiovascular disease and cancers [4,8-10]. Post-cholecystectomy constant inflow of bile into the intestine and its metabolites can directly affect the intestinal microbiota [11], causing shifts in the gut-brain and gut-muscle axes and thus indirectly affecting the etiology and course of many related diseases and disorders. Although it is not yet possible to predict how particular perturbations will modify the microbiota, it is possible that different microbiome configurations might allow stratified treatment and diet recommendations in the future, 
becoming a novel and powerful candidate for personalized treatment of human diseases [4]. Among more than 500 oral drugs tested, $13 \%$ were discovered to be metabolized by the microbiome [12]. Another study identified 30 human gut microbiome-encoded enzymes responsible for the biotransformation of 20 drugs to 59 candidate metabolites [13]. Such findings strongly suggest the importance of including microbiomes into the framework of precision medicine. Although the pathogenesis of cholesterol gallstones is still not fully understood, gut microbiota dysbiosis plays an important role in their formation [14]. There is a paucity of studies describing fecal/gut bacteriobiome profiles in GSD-afflicted patients, both before and after surgery. The aim of our study was to inventory the fecal microbiota composition, as assessed by $16 \mathrm{~S}$ rRNA gene sequencing, in a cohort of female patients with GSD and compare bacterial diversity before and after CCE.

\section{Materials and Methods}

\subsection{Participants}

Twenty-eight female patients with gallstone disease diagnosed by abdominal ultrasonography were recruited for the study (Table 1). The older patients had a higher BMI (Pearson's correlation coefficient $0.66, p<0.001$ ). All patients underwent clinical examination to assess their gastrointestinal and gallbladder status and severity of their clinical condition; 21 patients had chronic disease, and the rest had acute disease. The patients had no history of treatment with antibiotics and proton pump inhibitors at least for 1 month prior to feces sampling, as well as no probiotics and/or prebiotics as special supplementation. Half of the patients had arterial hypertension, associated with increased BMI. The patients fasted for at least $12 \mathrm{~h}$ before the surgery. After the surgery, the patients received the antibiotic ceftriaxone. No specific diet was prescribed after the surgery.

Table 1. Demographics of the study cohort $(\mathrm{N}=28$, females).

\begin{tabular}{ccccc}
\hline & Mean & Median & Min & Max \\
\hline Age, years & 51.6 & 55.0 & 18.0 & 73.0 \\
BMI ${ }^{\$}, \mathrm{~kg} / \mathrm{m}^{2}$ & 25.7 & 24.8 & 17.6 & 34.5 \\
\hline
\end{tabular}

$\$$ BMI stands for body mass index.

All patients were duly informed, gave their consent to the study and signed the informed consent form. The study observed all the relevant institutional and governmental regulations. The protocol of the study was approved by the Ethic Committee of the Research Institute of Internal and Preventive Medicine-Branch of the Institute of Cytology and Genetics, SB RAS. All clinical aspects of the study were supervised by a gastroenterologist.

\subsection{Fecal and Blood Sample Collection}

Fecal samples were collected 1 day prior to the CCE and 1-3 days after the surgery, i.e., as soon as patients had stool, into $10 \mathrm{~mL}$ sterile fecal specimen containers and stored at $-80{ }^{\circ} \mathrm{C}$ until use for DNA extraction. Blood samples were taken twice on the same day as stool samples.

\subsection{Blood Analyses}

Collected blood samples were used to determine aspartate aminotransferase (AST, EC 2.6.1.1) and alanine aminotransferase (ALT, EC 2.6.1.2) by the kinetic method, as recommended by the International Federation of Clinical Chemistry and Laboratory Medicine (IFCC2), using a biochemical analyzer, "Konelab Prime 30i" (Thermo Fisher Scientific, Vantaa, Finland).

\subsection{Extraction of Total Nucleic Acid from Feces}

Total DNA was extracted from 50 to $100 \mathrm{mg}$ of thawed patient fecal samples using the MetaHIT protocol [15]. The bead beating was performed using TissueLyser II (Qiagen, 
Hilden, Germany), for $10 \mathrm{~min}$ at $30 \mathrm{~Hz}$. No further purification of the DNA was needed. The quality of the DNA was assessed using agarose gel electrophoresis. No further purification of the DNA was needed.

\section{5. $16 S$ rRNA Gene Amplification and Sequencing}

The 16S rRNA genes were amplified with the primer pair V3/V4, combined with Illumina adapter sequences [16]. PCR amplification was performed as described earlier [17]. A total of $200 \mathrm{ng}$ of PCR product from each sample was pooled together and purified through MinElute Gel Extraction Kit (Qiagen, Hilden, Germany). The obtained amplicon libraries were sequenced with $2 \times 300$ bp paired-end reagents on MiSeq (Illumina, San Diego, USA) in the SB RAS Genomics Core Facility (ICBFM SB RAS, Novosibirsk, Russia). The read data reported in this study were submitted to GenBank under the study accession number PRJNA687360.

\subsection{Bioinformatic and Statistical Analyses}

Raw sequences were analyzed with the UPARSE pipeline [18] using Usearch v.11.0.667. The UPARSE pipeline included merging of paired reads; read quality filtering (-fastq_maxee_rate 0.005); length trimming (remove less than $350 \mathrm{nt}$ ); merging of identical reads (dereplication); discarding singleton reads; removing chimeras and OTU clustering using the UPARSEOTU algorithm. The OTU sequences were assigned a taxonomy using SINTAX [19] on the RDP database. As a reference for bacteria, we used the 16S RDP training set v.16 [20]. Statistical analyses (descriptive statistics, Wilcoxon's test for dependent variables, principal component analysis, multiple regression and general linear model, GLM, analysis with repeated measures) were performed by using Statistica v.13.3. Principle coordinate analysis (PCoA) was performed by PAST software v.3.17 [21]. The individual rarefaction showed that the sampling effort reached saturation for all samples (Figure S1); therefore, $\alpha$ biodiversity indices were calculated for complete datasets using PAST software v.3.17 [21]. Statistical significance was defined as $p<0.05$.

\section{Results}

\subsection{Overall Bacteriobiome Diversity}

After quality filtering and chimera and non-bacterial sequence removal, a total of 916 OTUs were identified at the $97 \%$ sequence identity level. All these OTUs could not be ascribed to a species level. Overall, they clustered into 11 phyla, 24 classes (with 18 explicitly classified at the class level), 59 orders (with 50 explicitly classified at the order level), 72 families (with 54 explicitly classified at the family level) and 172 genera (with 132 explicitly classified at the genus level). Sixty-one OTUs, i.e., ca. 7\% of the species richness and ca. $0.1 \%$ of the relative abundance, could not be ascribed below the domain level. Firmicutes with 635 OTUs was, by far, the most species-rich phylum, accounting for $69 \%$ of the total number of OTUs. Bacteroidetes ranked second richest with 96 OTUs (10.5\%), followed by Actinobacteria (64 OTUs, 7\%) and Proteobacteria with 45 (5\%). Such phyla as Synergistetes, Tenericutes and Verrucomicrobia were represented by 3-5 OTUs each, whereas the rest of the identified phyla, i.e., Spirochaetes, Fusobacteria, Lentisphaerae and cand. Saccharibacteria, were represented by one OTU each. The Firmicutes phylum was also the ultimate dominant phylum, accounting, on average, for ca. $66 \%$ of the total number of sequence reads. The Firmicutes/Bacteroidetes ratio varied widely: from 0.4 to 6837 (median 5.0) before CCE and from 0.4 to 2918 (median 3.8) after CCE (Table S1), showing no CCErelated difference ( $p=0.39$, Wilcoxon's test) and no correlation with blood biochemistry (Spearman's, $p>0.05$ ).

The dominant bacterial OTUs, i.e., OTUs contributing $\geq 1 \%$ (mean abundance) to the total number of sequence reads obtained for a sample, amounted to 27, with 18 OTUs representing Firmicutes, and Bacteroidetes and Actinobacteria contributing four and three OTUs, respectively, whereas Veruccomicrobia and Synergestetes each contributed one OTU to 
the dominants' pool. Thus, the overwhelming majority (ca. 97\%) of the OTUs in the study were minor or rare species.

\subsection{Fecal Bacteriobiome Composition in GSD Patients before the CCE Surgery}

The fecal microbiota of GSD patients was dominated by the Firmicutes phylum $(66 \%$ relative abundance), followed by Bacteroidetes (19\%), Actinobacteria $(8 \%)$ and Proteobacteria (4\%) phyla (Figure 1a). At the class level, the ultimate dominance of Firmicutes translated into the dominance of its classes Clostridia (47\%), Bacilli (11\%), Negativicutes (5\%) and Erysipelotrichia (1.4\%). Bacteroidetes was represented by the Bacteroidia class (19\%), whereas Actinobacteria was represented solely by the Actinobacteria class. Proteobacteria was present mostly as Gammaproteobacteria, with Alpha-, Beta-, Delta- and Epsilonproteobacteria summarily contributing less than $1 \%$. As for orders, Clostridiales, Lactobacillales, Selenomonadales and Erysipelotrichales (Firmicutes), Bacteroidales (Bacteroidetes), Bifidobacteriales and Coriobacteriales (Actinobacteria) and Enterobacteriales (Gammaproteobacteria) were found to prevail. Just three Firmicutes families, namely, Ruminococcaceae, Lachnospiraceae and Enterococcaceae, together accounted for half of the bacteriobiome abundance (Figure 1b). Most of the dominant OTUs (Figure 1c) belonged to the genera of the abovementioned families, i.e., Enerococcus, Gemmiger, Faecalibacterium, Ruminococcus, Blautia, Roseburia and Streptococcus. Other dominant OTUs represented Bacteroidetes/Bacteroidia/Bacteroidales (Prevotella sp. and Bacteroides sp.) and Bifidobacteriaceae/Bifidobacteriales/Actinobacteria (Bifidobacterium sp.).

Overall, fecal bacterial assemblages of GSD-afflicted subjects were characterized by high inter-individual variability of relative abundance and many outliers or extreme values at all taxonomic levels (Figure 1). Since we could not reasonably explain the outliers by errors in sampling collection and handling, nor by patients' characteristics and analytical procedures, we performed principal component (PC) analysis (based on covariance) of the data matrix with bacterial relative abundances as variables for analysis and patients as subjects in order to (a) obtain a better insight into the variance structure throughout the cohort, (b) find an association of the major PCs with patients' demographics and blood characteristics and then (c) implicate some bacterial taxa that contributed the most to the major PCs, as major players in such associations.

PC and multiple regression analyses showed that the core phyla, accounting for most of the data variance, showed a tendency for some association (PC2) with age, whereas some minor dominants (PC3, PC4) showed a correlation with blood glucose and bilirubin (Table 2). 
Table 2. Statistical analyses' results: contribution of bacterial phyla to the principal components extracted from the matrix with relative abundance in feces of females with GSD before CCE (percentage of the total data variance in brackets) and $p$-values for multiple regression with age, BMI and blood biochemistry.

\begin{tabular}{|c|c|c|c|c|}
\hline \multicolumn{5}{|c|}{ Phyla: PCA ${ }^{1}$} \\
\hline Main & PC 1 & PC 2 & PC 3 & PC 4 \\
\hline contributors & $(65 \%)$ & $(18 \%)$ & $(11 \%)$ & $(5 \%)$ \\
\hline Bacteroidetes & $0.49^{2}[-0.96]^{3}$ & $0.29[-0.23]$ & 0.04 & 0.01 \\
\hline Firmicutes & $\mathbf{0 . 4 8}[0.91]$ & $0.29[-0.39]$ & 0.00 & 0.06 \\
\hline Proteobacteria & 0.01 & 0.14 & $\mathbf{0 . 2 5}[0.03]$ & $\mathbf{0 . 1 4}[0.98]$ \\
\hline Actinobacteria & 0.01 & $0.23[0.97]$ & $0.59[-0.18]$ & 0.00 \\
\hline Verrucomicrobia & 0.00 & 0.05 & 0.04 & $0.78[-0.13]$ \\
\hline \multicolumn{5}{|c|}{ Phyla: Multiple regression } \\
\hline $\mathrm{R} / \mathrm{R}^{2}$ & $0.54 / 0.29$ & $0.53 / 0.28$ & $0.61 / 0.37$ & $0.57 / 0.33$ \\
\hline Age & 0.70 & $\underline{0.09}^{4}$ & 0.38 & 0.16 \\
\hline BMI & 0.39 & 0.21 & 0.37 & 0.21 \\
\hline Glucose & 0.13 & 1.00 & $\underline{0.07}$ & 0.70 \\
\hline ALT & 0.84 & 0.68 & $\overline{0.11}$ & 0.12 \\
\hline AST & 0.51 & 0.61 & 0.80 & 0.17 \\
\hline Bilirubin & 0.13 & 0.20 & 0.70 & $\underline{0.10}$ \\
\hline
\end{tabular}

1 PCA stands for principle component analysis (based on covariance). Only those principal components that (a) account for the bigger fraction of the total data variance and/or (b) displayed a statistically significant correlation with patients' characteristics are shown. ${ }^{2}$ The values in bold show the two topmost contributions. ${ }^{3}$ Factor loadings for variables (taxon relative abundance) are given in square brackets. ${ }^{4}$ The values in bold italics and underlined italics are at $p \leq 0.05$ and $p \leq 0.10$, respectively. 


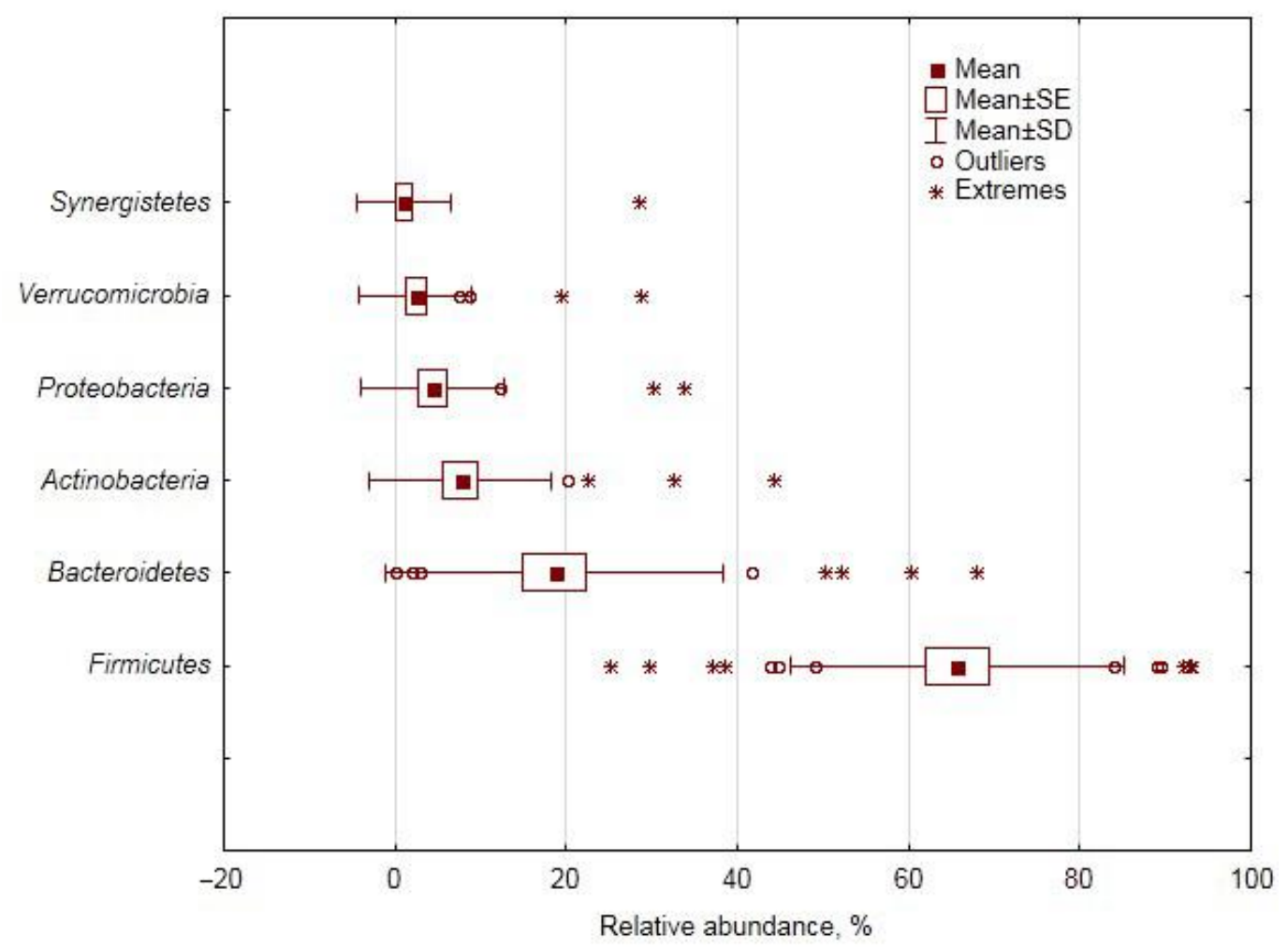

(a)

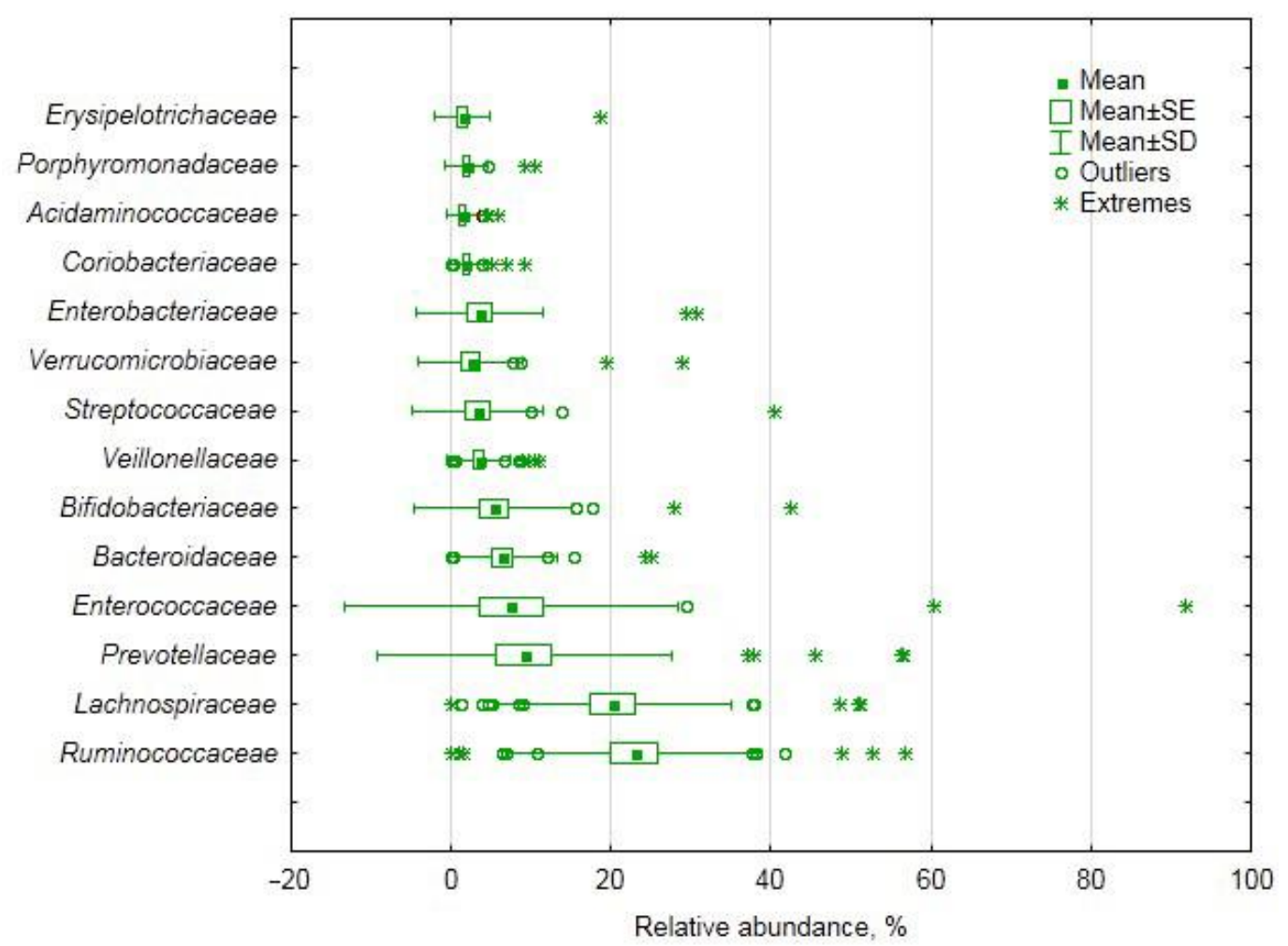

(b)

Figure 1. Cont. 


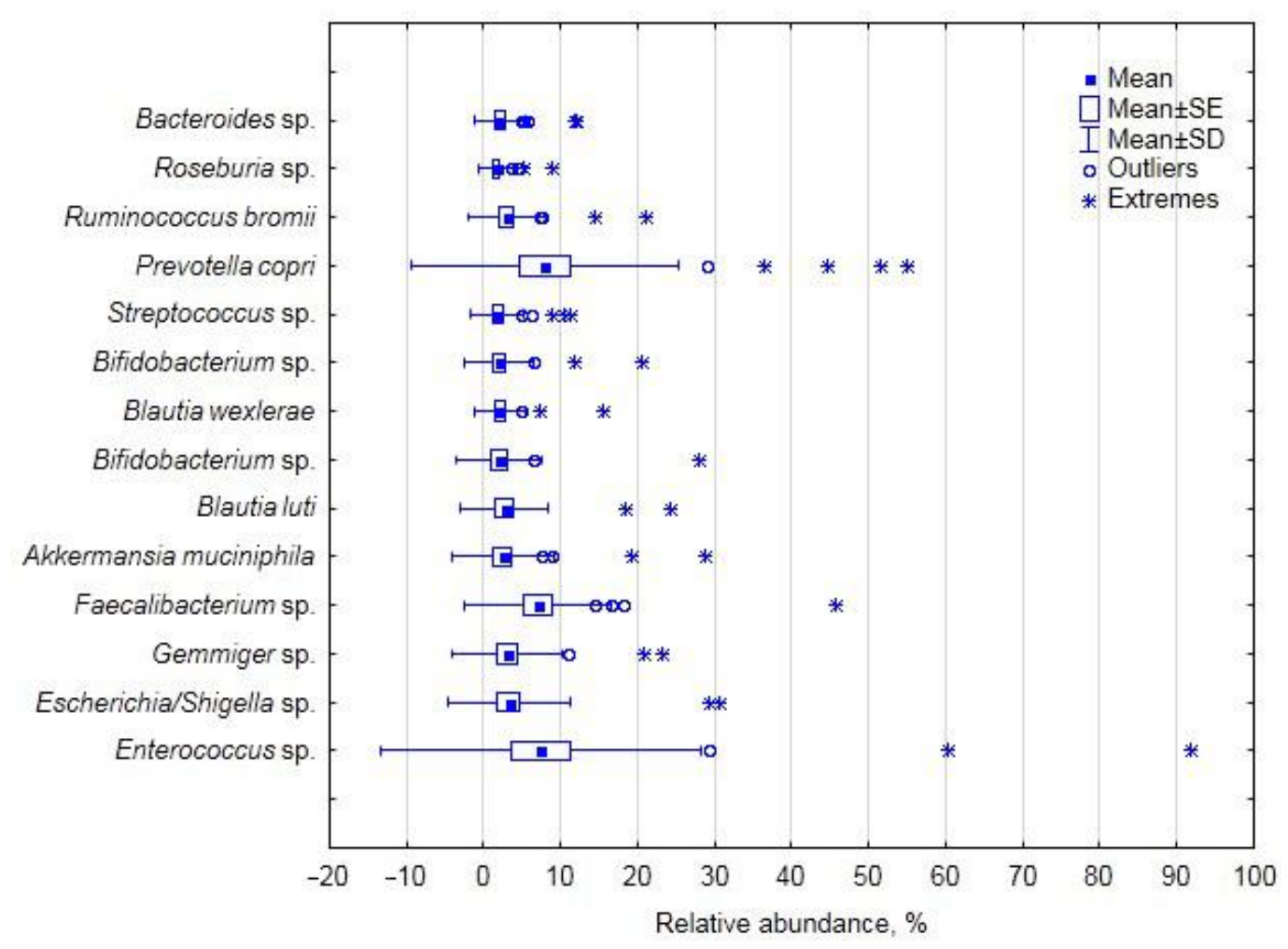

(c)

Figure 1. Relative abundance of the dominant bacterial taxa in females with gallstone disease (GSD) before cholecystectomy (CCE): (a) phyla, (b) families, (c) operational taxonomic units (OTUs).

As for the classes, the balance between the two core ones, i.e., Clostridia and Bacteroidia (PC2), was correlated with blood glucose, whereas the balance between two minor dominants, i.e., Actinobacteria and Gammaproteobacteria (PC4), was correlated with age (Table S2).

The core orders, i.e., Clostridiales and Lactobacillales, both belonging to the different classes of the Firmicutes phylum, accounted for half of the data variance at this taxonomical level, showing some age correlation tendency (Table S3).

At the family level, the balance between Ruminococcaceae and Enterococcaceae (PC1), both representing the ultimately dominating Firmicutes phylum, correlated strongly with the age and BMI of the studied cohort, whereas a tiny portion of the data variance (PC10), structured by the balance between Veillonellaceae and Erysipelotrichaceae, both also belonging to Firmicutes, was found to be associated with age, glucose and transaminase activity (Table S4).

At the genus level, the relative abundance was structured mainly by the balance between Prevotella (Bacteroidetes) and Enterococcus (Firmicutes), PC1 showing a correlation with blood bilirubin (Table 3). The balance between Faecalibacterium and Bifidobacterium showed some association with glucose, whereas the relationship between Blautia and some unclassified genus of the Ruminococcaceae family (PC6) had a statistically significant correlation with blood bilirubin and transaminase activity (Table 3).

As for the species level, the major part of the relative abundance variance was accounted for by the relationship between Enterococcus sp. (Firmicutes) and Prevotella copri (Bacteroidetes), correlating with age, BMI and, possibly, blood glucose (Table 4), whereas small portions of the data variance, attributed to the balance between Blautia luti (Firmicutes) and Akkermansia muciniphila (PC6) and between two Bifidobacterium OTUs (PC8), could be partially ascribed to blood bilirubin and transaminase activity (Table 4). 
Table 3. Statistical analyses' results: contribution of bacterial genera to the principal components extracted from the matrix with relative abundance in feces of females with gallstone disease before CCE (percentage of total variance in brackets) and $p$-values for multiple regression with blood biochemistry (coefficients of determination in brackets).

\begin{tabular}{|c|c|c|c|c|}
\hline \multicolumn{5}{|c|}{ Genera: PCA ${ }^{1}$} \\
\hline Main & PC 1 & PC 2 & PC 5 & PC 6 \\
\hline contributors & $(35 \%)$ & $(21 \%)$ & $(5 \%)$ & $(5 \%)$ \\
\hline Prevotella & $\mathbf{0 . 1 0}{ }^{2}[0.41]^{3}$ & $0.79[-0.90]$ & 0.00 & 0.00 \\
\hline Enterococcus & $0.84[-0.97]$ & $\mathbf{0 . 0 7}[0.22]$ & 0.01 & 0.00 \\
\hline Faecalibacterium & 0.02 & 0.00 & $0.17[-0.37]$ & 0.02 \\
\hline Blautia & 0.00 & 0.03 & 0.01 & $0.21[0.50]$ \\
\hline Bifidobacterium & 0.01 & 0.04 & $\mathbf{0 . 3 0}[-0.49]$ & 0.00 \\
\hline Gemmiger & 0.00 & 0.02 & $0.15[0.45]$ & 0.00 \\
\hline Ruminococcus & 0.00 & 0.01 & 0.01 & $0.07[-0.39]$ \\
\hline un. Ruminococcaceae & 0.00 & 0.01 & 0.01 & $0.21[-0.57]$ \\
\hline \multicolumn{5}{|c|}{ Genera: Multiple regression } \\
\hline $\mathrm{R} / \mathrm{R}^{2}$ & $0.72 / 0.51$ & $0.56 / 0.32$ & $0.64 / 0.41$ & $0.71 / 0.50$ \\
\hline Age & 0.16 & 0.92 & 0.13 & 0.12 \\
\hline BMI & 0.29 & 0.72 & $0.09^{3}$ & 0.42 \\
\hline Glucose & 0.71 & 0.25 & $\overline{0.03^{4}}$ & 0.76 \\
\hline ALT & 0.55 & 0.22 & 0.12 & 0.03 \\
\hline AST & 0.73 & 0.48 & 0.10 & 0.03 \\
\hline Bilirubin & 0.04 & 0.55 & $\overline{0.17}$ & 0.04 \\
\hline
\end{tabular}

${ }_{1}^{1}$ PCA stands for principle component analysis (based on covariance). Only those principal components that (a) account for the bigger fraction of the total data variance and/or (b) displayed a statistically significant correlation with patients' characteristics are shown. ${ }^{2}$ The values in bold show the two topmost contributions. ${ }^{3}$ Factor loadings for variables (taxon relative abundance) are given in square brackets. ${ }^{4}$ The values in bold italics and underlined italics are at $p \leq 0.05$ and $p \leq 0.10$, respectively.

Table 4. Statistical analyses' results: contribution of bacterial OTUs into the principal components extracted from the matrix with relative abundance in feces of females with gallstone disease before CCE (percentage of total variance in brackets) and $p$-values for multiple regression with age, BMI and blood biochemistry (coefficients of determination in brackets).

\begin{tabular}{|c|c|c|c|c|c|c|c|}
\hline \multicolumn{8}{|c|}{ OTUs: PCA ${ }^{1}$} \\
\hline Main & PC 1 & PC 3 & PC 4 & & PC 6 & & PC 8 \\
\hline contributors & $(40 \%)$ & $(9 \%)$ & $(7 \%)$ & & $(3 \%)$ & & $(2 \%)$ \\
\hline Enterococcus sp. & $0.86^{2}[0.96]^{3}$ & 0.00 & 0.00 & & 0.00 & & 0.00 \\
\hline Escherichia/Shigella sp. & 0.00 & $\begin{array}{c}\mathbf{0 . 2 5} \\
{[-0.65]}\end{array}$ & $0.25[-0.57]$ & & 0.07 & & 0.01 \\
\hline Gemmiger & 0.00 & 0.01 & $0.28[0.67]$ & & 0.00 & & 0.00 \\
\hline Faecalibacterium prausnitzii & 0.01 & $\mathbf{0 . 6 3}[0.86]$ & $0.16[-0.38]$ & & 0.06 & & 0.02 \\
\hline Akkermansia muciniphila & 0.00 & 0.02 & $0.09[0.41]$ & & $0.22[-0.45]$ & & 0.09 \\
\hline Blautia luti & 0.00 & 0.01 & 0.05 & & $0.26[-0.56]$ & & 0.02 \\
\hline Bifidobacterium sp. & 0.00 & 0.03 & 0.02 & & $0.07[-0.30]$ & & $0.35[-0.49]$ \\
\hline Bifidobacterium sp. & 0.00 & 0.00 & 0.00 & & $0.19[0.60]$ & & $0.27[-0.52]$ \\
\hline Streptococcus sp. & 0.00 & 0.00 & 0.00 & & 0.02 & & $0.13[0.48]$ \\
\hline Prevotella copri & $0.12[-0.42]$ & 0.00 & 0.00 & & 0.01 & & 0.00 \\
\hline \multicolumn{8}{|c|}{ OTUs: Multiple regression } \\
\hline $\mathrm{R} / \mathrm{R}^{2}$ & $0.71 / 0.50$ & 0.3 & & $0.40 / 0.16$ & & $0.73 / 0.53$ & $0.54 / 0.29$ \\
\hline Age & $0.05^{4}$ & & & 0.34 & & 0.55 & 0.11 \\
\hline BMI & 0.03 & & & 0.92 & & $\underline{0.09}$ & 0.35 \\
\hline Glucose & $\underline{0.07^{3}}$ & & & $\underline{0.07}$ & & 0.39 & 0.76 \\
\hline ALT & 0.52 & & & 0.92 & & 0.00 & 0.03 \\
\hline AST & 0.68 & & & 0.98 & & 0.00 & 0.03 \\
\hline Bilirubin & 0.16 & & & 0.64 & & 0.02 & 0.05 \\
\hline
\end{tabular}

${ }^{1}$ PCA stands for principle component analysis (based on covariance). Only those principal components that (a) account for the bigger fraction of the total data variance and/or (b) displayed a statistically significant correlation with patients' characteristics are shown. ${ }^{2}$ The values in bold show the two topmost contributions. ${ }^{3}$ Factor loadings for variables (taxon relative abundance) are given in square brackets. ${ }^{4}$ The values in bold italics and underlined italics are at $p \leq 0.05$ and $p \leq 0.10$, respectively. 


\subsection{Changes in Fecal Bacteriobiome Composition in GSD Patients after the CCE Surgery}

At the phylum level, CCE did not show any effect, whereas an effect was revealed for the Clostridia class and the Clostridiales and Coriobacteriales orders (Table 5). Further down the taxonomical hierarchy, the effect was displayed by the differential surgeryrelated abundance of the Clostridiaceae_1,_Lachnospiraceae and Peptoniphilaceae families (all belonging to Clostridiales) and Coriobacteriaceae of the namesake order of the Actinobacteria phylum (Table 6). Lachnospiraceae, being the predominating family with 129 OTUs in the studied cohort, accounted for $20 \%$ of the total number of Firmicutes OTUs and ranked the top family in abundance (with ca. 20\%); its decreased post-CCE abundance was manifested by Blautia, Roseburia and some unclassified representatives of the family at the genus level (Table 6).

Table 5. The relative abundance of some higher bacterial taxa in patients' feces before and after CCE.

\begin{tabular}{|c|c|c|c|c|c|}
\hline & \multicolumn{2}{|c|}{ Before CCE } & \multicolumn{2}{|c|}{ After CCE } & \multirow{2}{*}{$p$-Value } \\
\hline & Median & Mean \pm SD & Median & $\begin{array}{c}\text { Mean } \pm \\
\text { SD }\end{array}$ & \\
\hline \multicolumn{6}{|c|}{ Phyla (dominant) } \\
\hline Firmicutes & 66.7 & $65.7 \pm 19.6$ & 64.7 & $65.4 \pm 21.4$ & 0.34 \\
\hline Bacteroidetes & 12.2 & $18.6 \pm 19.8$ & 11.0 & $19.2 \pm 21.4$ & 0.53 \\
\hline Actinobacteria & 3.8 & $7.7 \pm 10.6$ & 4.0 & $7.2 \pm 9.8$ & 0.96 \\
\hline Proteobacteria & 0.5 & $4.4 \pm 8.4$ & 0.8 & $2.8 \pm 4.9$ & 0.55 \\
\hline Verrucomicrobia & 0.0 & $2.4 \pm 6.6$ & 0.0 & $3.8 \pm 9.4$ & 0.09 \\
\hline un. ${ }^{1}$ Bacteria & 0.1 & $0.14 \pm 0.27$ & 0.1 & $0.12 \pm 0.23$ & 0.20 \\
\hline \multicolumn{6}{|c|}{ Classes (dominant) } \\
\hline Clostridia $^{2}$ & 49.3 & $46.8 \pm 23.1$ & 42.9 & $40.7 \pm 23.1$ & 0.01 \\
\hline Bacteroidia & 12.2 & $18.6 \pm 19.8$ & 10.9 & $19.2 \pm 21.4$ & 0.47 \\
\hline Bacilli & 1.2 & $11.1 \pm 23.0$ & 0.5 & $16.8 \pm 30.0$ & 0.51 \\
\hline Actinobacteria & 3.8 & $7.7 \pm 10.6$ & 4.0 & $7.2 \pm 9.8$ & 0.97 \\
\hline Negativicutes & 4.5 & $4.9 \pm 3.9$ & 3.0 & $5.2 \pm 6.3$ & 0.84 \\
\hline Verrucomicrobiae & 0.0 & $2.4 \pm 6.6$ & 0.0 & $3.8 \pm 9.4$ & 0.09 \\
\hline Gammaproteobacteria & 0.0 & $3.5 \pm 8.2$ & 0.0 & $2.1 \pm 5.0$ & 0.43 \\
\hline un. Firmicutes & 0.3 & $1.5 \pm 3.2$ & 0.5 & $1.4 \pm 1.9$ & 0.70 \\
\hline Erysipelotrichia & 0.5 & $1.4 \pm 3.5$ & 0.4 & $1.4 \pm 2.6$ & 0.29 \\
\hline \multicolumn{6}{|c|}{ Orders (dominant) } \\
\hline Clostridiales & 43.3 & $44.8 \pm 23.5$ & 40.5 & $39.0 \pm 23.2$ & 0.01 \\
\hline Bacteroidales & 12.5 & $20.4 \pm 20.4$ & 14.6 & $21.3 \pm 22.2$ & 0.47 \\
\hline Lactobacillales & 1.2 & $12.0 \pm 22.5$ & 0.9 & $16.9 \pm 28.9$ & 0.50 \\
\hline Selenomonadales & 4.5 & $5.2 \pm 4.5$ & 3.2 & $5.7 \pm 6.7$ & 0.84 \\
\hline Bifidobacteriales & 0.7 & $4.9 \pm 9.6$ & 0.0 & $4.4 \pm 8.8$ & 0.53 \\
\hline Verrucomicrobiales & 0.0 & $2.3 \pm 6.4$ & 0.0 & $3.5 \pm 9.1$ & 0.09 \\
\hline Enterobacteriales & 0.0 & $3.3 \pm 7.7$ & 0.0 & $1.9 \pm 4.7$ & 0.39 \\
\hline Coriobacteriales & 0.8 & $1.9 \pm 2.2$ & 1.0 & $2.4 \pm 2.7$ & 0.03 \\
\hline Erysipelotrichales & 0.5 & $1.4 \pm 3.4$ & 0.4 & $1.3 \pm 2.5$ & 0.25 \\
\hline
\end{tabular}

${ }^{1}$ un. stands for unclassified. ${ }^{2}$ Gray-shadowed lines have $p$-values $\leq 0.05$. 
Table 6. The relative abundance of some bacterial families, genera and OTUs in patients' feces before and after CCE.

\begin{tabular}{|c|c|c|c|c|c|}
\hline \multirow{2}{*}{ Taxon } & \multicolumn{2}{|c|}{ Before CCE } & \multicolumn{2}{|c|}{ After CCE } & \multirow{2}{*}{$p$-Value } \\
\hline & Median & Mean \pm SD & Median & Mean \pm SD & \\
\hline \multicolumn{6}{|c|}{ Families } \\
\hline Clostridiaceae_1 4 & & $0.2 \pm 0.4$ & & $0.3 \pm 0.5$ & 0.010 \\
\hline Lachnospiraceae & & $20.3 \pm 14.8$ & & $16.6 \pm 14.0$ & 0.032 \\
\hline Coriobacteriaceae & & $1.9 \pm 2.3$ & & $2.4 \pm 2.8$ & 0.033 \\
\hline Peptoniphilaceae & & $0.005 \pm 0.013$ & & $0.002 \pm 0.007$ & 0.036 \\
\hline Peptostreptococcaceae & & $0.4 \pm 0.6$ & & $0.6 \pm 1.0$ & 0.054 \\
\hline Ruminococcaceae & & $22.9 \pm 15.9$ & & $19.5 \pm 13.8$ & 0.056 \\
\hline Rhodospirillaceae & & $0.1 \pm 0.4$ & & $0.01 \pm 0.05$ & 0.059 \\
\hline \multicolumn{6}{|c|}{ Genera } \\
\hline Clostridium s.s. & & $0.2 \pm 0.4$ & & $0.3 \pm 0.5$ & 0.010 \\
\hline Gordonibacter & & $0.01 \pm 0.04$ & & $0.01 \pm 0.07$ & 0.036 \\
\hline Peptoniphilus & & $0.005 \pm 0.013$ & & $0.002 \pm 0.007$ & 0.036 \\
\hline $\begin{array}{c}\text { un }^{1} \text {. } \\
\text { Rhodospirillaceae }\end{array}$ & & $0.08 \pm 0.36$ & & $0.01 \pm 0.05$ & 0.059 \\
\hline Gemmiger & & $4.3 \pm 7.4$ & & $3.2 \pm 4.8$ & 0.065 \\
\hline Collinsella & & $1.0 \pm 1.8$ & & $1.3 \pm 2.1$ & 0.066 \\
\hline Blautia & & $6.4 \pm 8.8$ & & $4.9 \pm 7.3$ & 0.067 \\
\hline Enterococcus & & $7.4 \pm 20.8$ & & $13.6 \pm 26.4$ & 0.080 \\
\hline Faecalibacterium & & $7.7 \pm 9.8$ & & $6.1 \pm 8.6$ & 0.089 \\
\hline Roseburia & & $2.8 \pm 4.9$ & & $1.6 \pm 2.5$ & 0.089 \\
\hline Dialister & & $1.5 \pm 2.5$ & & $1.0 \pm 2.1$ & 0.093 \\
\hline Peptostreptococcus & & $0.018 \pm 0.063$ & & $0.024 \pm 0.077$ & 0.093 \\
\hline \multicolumn{6}{|c|}{ OTUs } \\
\hline un.Lachnospiraceae & & $0.09 \pm 0.17$ & & $0.04 \pm 0.11$ & 0.003 \\
\hline un.Clostridium s.s. ${ }^{2}$ & & $0.14 \pm 0.43$ & & $0.3 \pm 0.5$ & 0.005 \\
\hline un.Clostridium XlVa & & $0.01 \pm 0.03$ & & $0.03 \pm 0.05$ & 0.021 \\
\hline un.Clostridiales & & $0.02 \pm 0.06$ & & $0.09 \pm 0.19$ & 0.023 \\
\hline Clostridium leptum & & $0.05 \pm 0.10$ & & $0.03 \pm 0.07$ & 0.025 \\
\hline un.Blautia & & $0.02 \pm 0.06$ & & $0.03 \pm 0.08$ & 0.029 \\
\hline Ruminococcus faecis & & $0.4 \pm 1.4$ & & $0.6 \pm 1.5$ & 0.030 \\
\hline un.Bacteroides & & $2.1 \pm 3.4$ & & $1.0 \pm 2.0$ & 0.033 \\
\hline Dialister invisus & & $1.0 \pm 2.1$ & & $0.4 \pm 1.0$ & 0.035 \\
\hline un.Ruminococcus & & $0.15 \pm 0.37$ & & $0.07 \pm 0.24$ & 0.036 \\
\hline un.Ruminococcus & & $0.2 \pm 0.6$ & & $0.07 \pm 0.29$ & 0.042 \\
\hline $\begin{array}{c}\text { un.Lachnospiracea } \\
\text { i.s. }{ }^{3}\end{array}$ & & $0.5 \pm 0.8$ & & $0.2 \pm 0.4$ & 0.050 \\
\hline un.Coriobacteriaceae & & $0.04 \pm 0.09$ & & $0.14 \pm 0.40$ & 0.059 \\
\hline un.Ruminococcaceae & & $0.09 \pm 0.16$ & & $0.18 \pm 0.30$ & 0.068 \\
\hline un.Ruminococcaceae & & $\begin{array}{c}0.0008 \pm \\
0.0017\end{array}$ & & $\begin{array}{c}0.0002 \pm \\
0.001\end{array}$ & 0.076 \\
\hline un.Enterococcus & & $7.4 \pm 20.8$ & & $13.6 \pm 26.4$ & 0.080 \\
\hline un.Lachnospiraceae & & $0.01 \pm 0.02$ & & $0.002 \pm 0.007$ & 0.080 \\
\hline un.Clostridiales & & $0.01 \pm 0.03$ & & $0.03 \pm 0.08$ & 0.083 \\
\hline un.Collinsella & & $1.0 \pm 1.8$ & & $1.3 \pm 2.1$ & 0.093 \\
\hline $\begin{array}{c}\text { Peptostreptococcus } \\
\text { stomatis }\end{array}$ & & $0.02 \pm 0.06$ & & $0.02 \pm 0.08$ & 0.093 \\
\hline
\end{tabular}

${ }^{1}$ un. stands for unclassified; ${ }^{2}$ s.s. stands for sensu stricto; ${ }^{3}$ i.s. stands for incertae sedis. ${ }^{4}$ Gray-shadowed lines have $p$-values $\leq 0.05$.

As for other genera, Clostridium sensu stricto increased, whereas Peptoniphilus decreased their presence in the fecal bacteriobiome of the studied cohort. Although Coriobacteriaceae increased their post-CCE abundance and were among the predominating families, at the genus level, they were represented by eight genera, only one of which (Gordonibacter) had a 
differential CCE-related abundance $(p \leq 0.05)$, albeit at the very low level, and Collinsella with its post-CCE increased abundance at the $p \leq 0.10$ level (Table 6). At the species level, 20 OTUs manifested surgery-related differences in their relative abundance at the $p \leq 0.10$ level of statistical significance, with 17 OTUs attributed to Firmicutes, two OTUs to Actinobacteria and one OTU to the Bacteroidetes phylum. Enterococcus sp. of Firmicutes was the leading dominant, increasing its abundance almost two-fold after the surgery (Table 6). Bacteroides sp. and Collinsella sp. were minor dominants with relative abundance around $1 \%$. The rest of the OTUs with differential CCE-related abundance were minor or rare species.

The results of GLM analysis with repeated measures (before and after CCE) and age and BMI as continuous factors (covariates) show that residuals complied with a normal distribution only for the Firmicutes taxa; nevertheless, this statistical approach revealed no CCE-associated effect on the phylum and its lower taxa abundance.

\subsection{Fecal Bacteriobiome $\alpha$-Diversity before and after the CCE Surgery}

No differences in $\alpha$-diversity indices at the $p \leq 0.05$ level were found in the studied cohort before and after CCE (Table 7). However, at the $p \leq 0.10$ level, evenness slightly decreased, whereas the maximal relative abundance (as shown by the Berger-Parker index) slightly increased. The location of samples in the plane of the first two principal coordinates (based on Bray-Curtis distance) did not reveal any distinct CCE-related pattern (Figure 2).

Table 7. Alpha-diversity indices estimated for patients' fecal bacterial assemblages before and after CCE.

\begin{tabular}{cccccc}
\hline \multirow{2}{*}{ Index } & \multicolumn{2}{c}{ Before CCE } & \multicolumn{2}{c}{ After CCE } & \multirow{2}{*}{$p$-Value } \\
\cline { 2 - 4 } & Median & Mean \pm SD & Median & Mean \pm SD & \\
\hline OTUs' richness & 90 & $93 \pm 41$ & 78 & $92 \pm 46$ & 0.89 \\
Chao-1 & 100 & $104 \pm 51$ & 85 & $104 \pm 55$ & 0.75 \\
Berger-Parker & 0.24 & $0.29 \pm 0.19$ & 0.27 & $0.33 \pm 0.21$ & 0.07 \\
Dominance (D) & 0.12 & $0.16 \pm 0.16$ & 0.12 & $0.19 \pm 0.17$ & 0.11 \\
Simpson (1-D) & 0.88 & $0.84 \pm 0.16$ & 0.88 & $0.81 \pm 0.17$ & 0.11 \\
Shannon & 2.8 & $2.8 \pm 0.8$ & 2.7 & $2.7 \pm 0.9$ & 0.21 \\
Evenness & 0.23 & $0.22 \pm 0.08$ & 0.20 & $0.20 \pm 0.09$ & 0.09 \\
Equitability & 0.67 & $0.63 \pm 0.13$ & 0.62 & $0.60 \pm 0.15$ & 0.13 \\
\hline
\end{tabular}

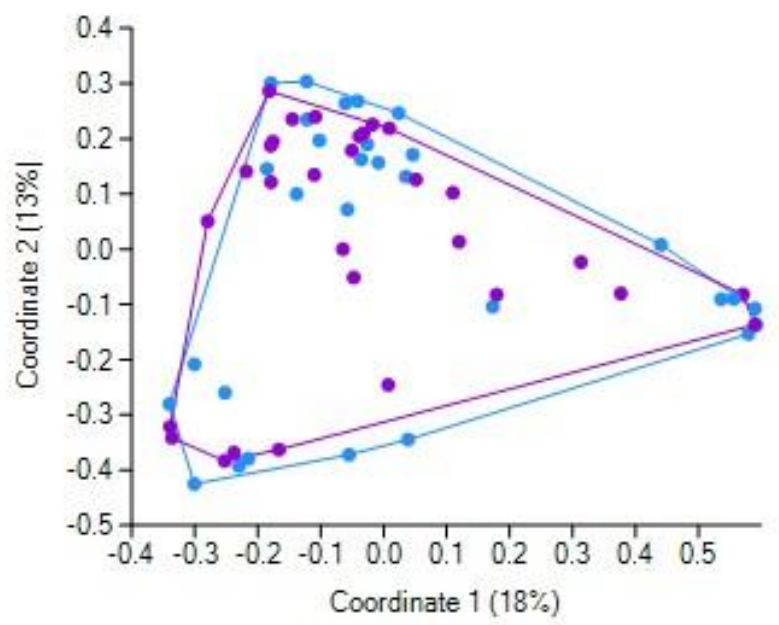

Figure 2. Location of patients' samples taken before (violet markers) and after (blue markers) CCE in the plane of the first two principal coordinates (Bray-Curtis distance). 


\subsection{Blood Biochemical Characteristics before and after the CCE Surgery}

After CCE, both aspartate and alanine transaminase activity mean values increased 1.7 and 1.6 times, with bilirubin levels being unaffected (Table 8). Age and BMI as continuous predictors in GLM analysis with repeated measures decreased the $p$-value for glucose content comparison $(p=0.06)$ while increasing it for direct bilirubin content $(p=0.80)$ (residuals for only these two dependent variables showed a normal distribution).

Table 8. Blood biochemical test results before and after CCE.

\begin{tabular}{cccccc}
\hline \multirow{2}{*}{ Property } & \multicolumn{2}{c}{ Before CCE } & \multicolumn{2}{c}{ After CCE } & p-Value \\
\cline { 2 - 5 } & Median & Mean \pm SD & Median & Mean \pm SD & \\
\hline $\begin{array}{c}\text { Glucose, mmol/L } \\
\text { Alanine }\end{array}$ & 5.25 & $5.24 \pm 0.88$ & 5.45 & $5.51 \pm 0.82$ & 0.107 \\
$\begin{array}{c}\text { transaminase, U/L } \\
\text { Aspartate }\end{array}$ & 20.2 & $36.0 \pm 68.5$ & 29.0 & $57.4 \pm 75.7$ & 0.001 \\
$\begin{array}{c}\text { transaminase, U/L } \\
\text { Bilirubin total, } \\
\text { mcmol/L }\end{array}$ & 19.9 & $34.4 \pm 47.9$ & 32.3 & $56.8 \pm 68.4$ & 0.001 \\
$\begin{array}{c}\text { Bilirubin direct, } \\
\text { mcmol/L } \\
\text { Bilirubin indirect, } \\
\text { mcmol/L }\end{array}$ & 11.8 & $18.8 \pm 21.1$ & 16.3 & $18.4 \pm 7.0$ & 0.168 \\
\hline
\end{tabular}

\section{Discussion}

\subsection{Fecal Bacteriobiome Composition in GSD Patients before the CCE Surgery}

In the studied cohort of GSD-afflicted female patients, the Firmicutes phylum was the ultimate dominant in the fecal bacteriobiome, with Bacteroidetes and Actinobacteria being second and third in the ranking of abundance. In this aspect, our cohort differed from a Chinese one, where Proteobacteria, instead of Actinobacteria, were found to be third in abundance, and Bacteroidetes were almost twice as abundant as in our cohort [22]. Moreover, in our cohort, the Firmicutes relative abundance was markedly higher, as compared with the Chinese cohorts $[5,21]$. The apparent discrepancy is most likely due to the fact that one third of the Chinese cohort were males, whereas ours was a purely female one; and to the difference in diet [23]. As for the biodiversity indices, however, the ones calculated in our study agree well with the indices describing the fecal bacteriobiome of a Chinese cohort of GSD patients [21].

High inter-individual variation in bacterial sequence reads is quite common in fecal bacteriobiome studies in cohorts of healthy human subjects and of those afflicted by various diseases, including GSD [21]. Even in cases when the authors [21] claimed that their results "showed that the individual differences within the group were small", the huge standard deviations for the OTUs' relative abundance in their study proved the opposite. Therefore, by structuring down the data variance in our study by principal component analysis, we identified some age- and BMI-related taxa within the studied cohort, as well as some taxa that showed a correlation with blood glucose, bilirubin and transaminase activity.

Some genera of Lachnospiraceae are known to be important for bile acid metabolism, having $7 \alpha$-dehydroxylation activity: in the pre-CCE bacteriobiome of the studied cohort, the family accounted for one fifth of the total number of sequence reads, along with Ruminocccaceae ultimately prevailing at the family level and together accounting for almost half of the abundance; in a Chinese cohort, however, the family with less than $0.3 \%$ was not even close to any dominating position [21]. The latter study also found the relative abundance of Clostridium to be $0.01 \%$, whereas in our study, the presence of Clostridium sensu stricto was an order of magnitude more pronounced (Table 6). The differences are plentiful and most likely attributable to racial, dietary, sex and other characteristics of the cohorts. 
Principal component analysis based on covariance allows dealing with the original variance of the data, without standardizing them, easily structuring the variance by extracted principal components, featuring the contribution of the original variables to the new ones (PCs) and reducing the original plethora of variables to fewer ones (PCs), but accounting for most of the original variance in the data. Subjection of the extracted PCs as dependent variables in multiple regression analysis allowed finding links with patients demographics and blood biochemical properties and bacterially interpreting them on the basis of a taxon contribution in the respective PC. We believe this approach to be informative for such kind of descriptive study.

The balance between two Firmicutes classes, namely, Clostridia and Bacilli, accounted for almost half of the abundance variance at this taxonomic level, being positively correlated with age at the $p \leq 0.10$ level; the situation was translated in a similar manner at the order level, i.e., Clostridiales and Lactobacillales. Then, at the family level, the balance between Ruminococcaceae (Clostridiales) and Enterococcaceae (Lactobacillales), accounting for one third of the total data variance (PC1), displayed a statistically significant positive correlation with age. This finding complies with the knowledge that the gut microbiota diversity changes with age [24,25], with Firmicutes taxa increasing. Thus, the increased abundance of the core gut bacterial taxa with age in GSD-compromised subjects seems quite a natural occurrence, not overshadowed by changed bile acid metabolism and other factors. Interestingly, at the genus level, the structure of the data variance shifted, with the Prevotella (Bacteroidetes) and Enterococcus (Firmicutes) relationship accounting for half of the total data variance at this taxonomic level; the finding underscores the importance of these two core taxa relationships in structuring the gut bacteriome in general and GSD-compromised female patients in particular. As increased plasma levels of bilirubin (secondary to the breakdown of free hemoglobin) were shown to be associated with an increased risk of gallstone disease [26], the statistically significant positive correlation of the Prevotella-Enterococcus balance with blood bilirubin, found in our study, necessitates further investigation of the role of the genera in gallstone formation, both pigmented and cholesterol ones [27].

The finding that the balance between Faecalibacterium, Bifidobacterium and Gemminer determined $10 \%$ of the total data variance and was correlated with blood glucose $(p \leq 0.05)$ and BMI $(p \leq 0.10)$ may be indicative of the indirect association of the genera with glucose metabolism and insulin sensitivity in overweight patients [28], but we cannot currently suggest the putative cause-effect mechanism. The joint variation in Blautia, Ruminococcus and some unclassified Ruminococcaceae correlated with blood ALT, AST and bilirubin, and the mechanism of the involvement of these genera has to be comprehensively examined.

Further down the hierarchy at the species level, the Prevotella-Enterococcus relationship was manifested by the balance between Prevotella copri and Enterococcus sp. (with $40 \%$ of the total data variance), which was positively correlated with the cohort's demographics, i.e., age and BMI $(p \leq 0.05)$, and glucose $(p \leq 0.10)$ and therefore may be related to glucose metabolism in overweight patients. Interestingly, Akkermansia muciniphila was found to contribute a small portion of the data variance associated with blood biochemistry (at $p \leq 0.05$ ) and, hence, generally with the disease-compromised status of the subjects. The increased relative abundance of this mucin-degrading bacterium is often found to be associated with disease $[29,30]$; however, as a propionogenic bacterium, A. muciniphila is also believed to have several health benefits in humans [31].

However, as the blood characteristics are far from being specific for gallstone disease diagnostics, it is not possible to implicate the taxa in the changed bile acid metabolism and gallstone formation based on the results of multiple regression analysis, despite the comprehensive outline of the GSD bacteriobiome variation as related to the common blood biochemical properties. The bile acid profiles of the studied patients, if they had been determined, might have been more suggestive in this respect, and we acknowledge this as a drawback in the study. 


\subsection{Changes in Fecal Bacteriobiome Composition in GSD Patients after the CCE Surgery}

In the studied cohort of GSD-afflicted female patients, mainly members of the Firmicutes phylum displayed CCE-related differential abundance.

As for the Bacteroidetes phylum, its relative abundance did not change after CCE; the result does not agree with the finding of Israeli researchers, for example, when the phylum abundance was shown to be increased in the post-CCE cohort of subjects [32]. However, the time factor, i.e., the duration of the time span elapsed between CCE and feces sampling, is a critical factor affecting bacterial composition [33] and may, at least partially, explain the discrepancy between results.

The decreased abundance of Clostridia (by 7\%) and Clostridiales (by 3-5\%), found after CCE in our study, was not reported before. As these taxa are the most species-rich and physiologically diverse components of the fecal bacteriobiome, the CCE-associated shifts in their relative abundance cannot be unequivocally regarded as beneficial or not for human health. Lachnospiraceae, the most predominant family in the human gut, displayed decreased abundance in the post-CCE bacteriobiome, which is, however, difficult to interpret as (a) most of the OTU-level clusters (69), ascribed to the family in our study (129), could not be taxonomically attributed below the family level, and (b) some genera and species of this family might support/contribute to healthy functions, whereas other genera and species were found to be increased in diseases [34]. For example, Blautia and Roseburia species, often associated with a healthy state, are some of the main short-chain fatty acid producers $[35,36]$; therefore, their post-CCE decreased abundance $(p \leq 0.10)$ may hardly be indicative of the better state of the gut bacteriobiome after surgery.

We could not find any information about the effect of CCE on Actinobacteria/ Coriobacteriales/Coriobacteriaceae representatives, the latter known as pathobionts. As for Collinsella, the dominant genus in the Coriobacteriaceae family and the minor dominant in the studied cohort, its increased abundance after CCE (albeit at the $p \leq 0.10$ level) suggests negative implications after such shift [37-40]. As for another representative of the family with differential pre- and post-CCE abundance, i.e., the Gordonibacter genus with just ca. $0.01 \%$ of the total number of sequence reads, it is difficult to suggest any ecophysiological significance at such abundance rate, although some genus representatives are known to participate in primary bile acid transformation [41] or be involved in dietary polyphenol transformations generating more bioactive metabolites [42].

Interestingly, although specific bacterial species such as Helicobacter and Salmonella were shown to be involved in the pathogenesis of cholesterol gallstones [43], we did not find Helicobacter at all, and found only one Salmonella OTU with 0.3 and $0.6 \%$ abundance in pre- and post-CCE subcohorts, respectively; the finding infers potentially different bacterial involvement in GSD etiology in cohorts of different sex and ethnicity.

The actual number of OTUs per sample observed in our study was practically the same as the number obtained by the same methodology for post-CCE patients in Korea [44]. However, as the latter study did not report whether the control group, i.e., non-CCE control patients, was also diagnosed with GSD, it is not possible to compare our results about the CCE effect on the fecal bacteriobiome with those results in their entirety, only for the post-CCE subcohort. For instance, in our study, CCE did not affect the gut bacteriobiome species richness, whereas compared with the independent control group, CCE decreased it [43]. Notably, the $\alpha$-biodiversity index (Shannon) reported in the aforementioned study was much higher than the one reported here ( 4.9 vs. 2.8$)$ : in our view, the discrepancy may be attributed to both the sex composition of the Korean cohort and the dietary habits, etc. At the same time, for the Israeli cohort of GDS patients, the pre- and post-CCE values of the Shannon index did not differ [31], being close to, but slightly lower than, those in our study ( 2.1 vs. 2.8 , respectively). We cannot help but emphasize that often the studies claiming to reveal the effect of CCE on the gut microbiota performed comparisons between the post-CCE patients with GSD and the healthy subjects without a GSD history $[33,43,44]$. We believe such approach does not seem to be adequate for aiming to examine the effect of CCE per se, as only a direct comparison between pre- and post-CCE conditions of one and 
the same cohort of GSD-affected subjects is pertinent for the goal of revealing microbiome shifts associated with the surgery and potential biomarkers of the latter.

It was shown that CCE did not markedly affect the bile acid profile in the GSD patients [31], leading the authors to conclude that the modified fecal bile acid composition results from inherently aberrant bile acid metabolism, leading, in turn, to gallstone formation. In general, our finding that the pre- and post-CCE fecal bacteriobiome profiles were not overall differentially distinct (as revealed by principal coordinate analysis) apparently complies with this conclusion. It should be emphasized that the repeated collection of feces samples was performed 1-3 days after the surgery, i.e., quite soon. Therefore, we are inclined to believe that it was a very short time to interpret the observed differential abundance of some bacterial taxa as solely resultant from the changed inflow of bile acids; the overall post-surgery condition most likely contributed significantly, if not primarily and predominantly, to the observed short-term CCE-related shifts in fecal bacterial assemblages. It should be noted that the overall post-surgery condition included ceftriaxone treatment of all patients. This beta-lactam antibiotic is able to kill a broad spectrum of bacteria [45], thus potentially shaping the gut bacteriobiome [46], especially when administered orally. Therefore, despite the very short time between the surgery and stool collection in this study, and hence the short time of antibiotic treatment, the revealed changes in the fecal bacteriobiome might have resulted, in part, due to the antibiotic per se. However, we should also emphasize that our study did not aim at discriminating between the effects of post-CCE altered bile profiles and antibiotic therapy; we aimed at profiling the gut bacteriobiome, referring to post-CCE as a single factor, as such embracing many factors, aspects, nuances, etc. We described the fecal bacteriobiome just at the starting point of patients embarking on the rest of life without a gallbladder. Whether the longer-term shifts in the gut microbiota after CCE will occur and to what degree and at what rate remain to be determined in future studies, which, hopefully, will also elucidate if gut microbes can act as the main character in the broad scenery of liver diseases [47].

\section{Conclusions}

Our study provides the first detailed inventory of the fecal bacteriobiome in a Russian cohort of female patients with gallstone disease. It will help to construct a global picture of the disease-related bacteriobiome and eventually focus on specific bacterial taxa involved in gallstone formation, thus facilitating the development of non-invasive therapeutic tools for preventing and treating gallstone disease. The shifts found in the fecal microbiota just a few days after CCE did not distinctly discriminate between the pre- and post-surgery bacterial diversity profiles. Therefore, the shifts can be mostly attributed to the surgery effect on the entire status of the patients, including the initial stages of the changing bile inflow and metabolism, as well as cellular and molecular modifications in the gut. The presented pre- and post-cholecystectomy microbiota profiles in one and the same cohort of patients may improve the insight into the relationship between the fecal, gut and bile microbiota, contributing to future larger-scale studies of altered human bile metabolism/profiles and associated disorders.

Supplementary Materials: The following are available online at https:/ / www.mdpi.com/article/ 10.3390/jpm11040294/s1, Figure S1: Rarefaction curves based on bacterial OTUs for the patients with gallstone disease before (a) and after (b) the cholecystectomy. The numbers in blue indicate patients' codes; Table S1: The ratio of Firmicutes/Bacteroidetes relative abundance in patients' feces before and after CCE.: Table S2: Statistical analyses' results: contribution of bacterial classes into the principal components extracted from the matrix with relative abundance in feces of females with gallstone disease before the CCE (percentage of total variance in brackets) and p-values for multiple regression with age, BMI and blood biochemistry; Table S3: Statistical analyses' results: contribution of bacterial orders into the principal components extracted from the matrix with relative abundance in feces of females with gallstone disease before the CCE (percentage of total variance in brackets) and p-values for multiple regression with age, BMI and blood biochemistry; Table S4: Statistical analyses' results: contribution of bacterial families into the principal components extracted 
from the matrix with relative abundance in feces of females with gallstone disease before the CCE (percentage of total variance in brackets) and p-values for multiple regression with age, BMI and blood biochemistry (coefficients of determination in brackets).

Author Contributions: Conceptualization, I.G.; methodology, I.G. and M.K.; software, M.K.; validation, M.K.; formal analysis, I.G. and T.A.; investigation, T.R. and A.K.; resources, I.G. and M.K.; data curation, N.N., T.R. and T.A.; writing-original draft preparation, N.N. and T.R.; writing-review and editing, I.G.; visualization, A.K.; supervision, I.G.; project administration, I.G. and M.K.; funding acquisition, I.G. All authors have read and agreed to the published version of the manuscript.

Funding: This research was funded by Russian State funded budget projects AAAA-A17-117112850280-2 and AAAA-A17-117020210021-7 with the financial support of the Biocodex MICROBIOTA Foundation, France.

Institutional Review Board Statement: The study was conducted according to the guidelines of the Declaration of Helsinki, and approved by the Institutional Review Board of the Research Institute of Internal and Preventive Medicine-Branch of the Institute of Cytology and Genetics SB RAS, Novosibirsk, Russia (protocol 38/1, approved 5 June 2018).

Informed Consent Statement: Informed consent was obtained from all subjects involved in the study.

Data Availability Statement: The read data reported in this study were submitted to GenBank under the study accession number PRJNA687360 (https:/ / www.ncbi.nlm.nih.gov / bioproject/?term= PRJNA687360).

Acknowledgments: The authors are very thankful to Semyon Chastnykh (Municipal Clinical Hospital No. 2, Novosibirsk, Russia) for his superb technical assistance.

Conflicts of Interest: The authors declare no conflict of interest. The funders had no role in the design of the study; in the collection, analyses, or interpretation of data; in the writing of the manuscript, or in the decision to publish the results.

\section{References}

1. Ilchenko, A.A. Diseases of the Gallbladder and Bile Ducts: Manual for Physicians, 2nd ed.; Medical Information Agency Publishers LLC: Moscow, Russia, 2011; 458p. (In Russian)

2. Vinnik, Y.S.; Serova, E.V.; Andreev, R.I.; Leyman, A.V.; Struzik, A.S. Conservative and surgical treatment of gallstone disease. Fudamental Res. 2013, 9, 954-958. (In Russian)

3. Grigor'eva, I.N.; Romanova, T.I. Gallstone Disease and Microbiome. Microorganisms 2020, 8, 835. [CrossRef] [PubMed]

4. Rezasoltani, S.; Sadeghi, A.; Radinnia, E.; Naseh, A.; Gholamrezaei, Z.; Azizmohammad Looha, M.; Yadegar, A. The association between gut microbiota, cholesterol gallstones, and colorectal cancer. Gastroenterol. Hepatol. Bed Bench 2019, 12 (Suppl. 1), S8-S13.

5. Wu, T.; Zhang, Z.; Liu, B.; Hou, D.; Liang, Y.; Zhang, J.; Shi, P. Gut microbiota dysbiosis and bacterial community assembly associated with cholesterol gallstones in large-scale study. BMC Genom. 2013, 14, 669. [CrossRef] [PubMed]

6. Wang, Q.; Jiao, L.; He, C.; Sun, H.; Cai, Q.; Han, T.; Hu, H. Alteration of gut microbiota in association with cholesterol gallstone formation in mice. BMC Gastroenterol. 2017, 17, 74. [CrossRef] [PubMed]

7. Chen, W.; Wei, Y.; Xiong, A.; Li, Y.; Guan, H.; Wang, Q.; Miao, Q.; Bian, Z.; Xiao, X.; Lian, M.; et al. Comprehensive Analysis of Serum and Fecal Bile Acid Profiles and Interaction with Gut Microbiota in Primary Biliary Cholangitis. Clin. Rev. Allergy Immunol. 2020, 58, 25-38. [CrossRef]

8. Di Ciaula, A.; Wang, D.Q.; Portincasa, P. Cholesterol cholelithiasis: Part of a systemic metabolic disease, prone to primary prevention. Expert Rev. Gastroenterol. Hepatol. 2019, 13, 157-171. [CrossRef]

9. Upala, S.; Sanguankeo, A.; Jaruvongvanich, V. Gallstone Disease and the Risk of Cardiovascular Disease: A Systematic Review and Meta-Analysis of Observational Studies. Scand. J. Surg. 2017, 106, 21-27. [CrossRef]

10. Fairfield, C.J.; Wigmore, S.J.; Harrison, E.M. Gallstone Disease and the Risk of Cardiovascular Disease. Sci. Rep. 2019, 9, 5830. [CrossRef]

11. Ramírez-Pérez, O.; Cruz-Ramón, V.; Chinchilla-López, P.; Méndez-Sánchez, N. The Role of the Gut Microbiota in Bile Acid Metabolism. Ann. Hepatol. 2017, 16 (Suppl. 1), s15-s20. [CrossRef]

12. Chankhamjon, P.; Javdan, B.; Lopez, J.; Hull, R.; Chatterjee, S.; Donia, M.S. Systematic mapping of drug metabolism by the human gut microbiome. bioRxiv 2019. bioRxiv:538215.

13. Zimmermann, M.; Zimmermann-Kogadeeva, M.; Wegmann, R.; Goodman, A.L. Mapping human microbiome drug metabolism by gut bacteria and their genes. Nature 2019, 570, 462-467. [CrossRef]

14. Grigor'eva, I.N. Gallstone Disease, Obesity and the Firmicutes/Bacteroidetes Ratio as a Possible Biomarker of Gut Dysbiosis. J. Pers. Med. 2021, 11, 13. [CrossRef] 
15. Godon, J.J.; Zumstein, E.; Dabert, P.; Habouzit, F.; Moletta, R. Molecular microbial diversity of an anaerobic digestor as determined by small-subunit rDNA sequence analysis. Appl. Environ. Microbiol. 1997, 63, 2802-2813. [CrossRef]

16. Fadrosh, D.W.; Ma, B.; Gajer, P.; Sengamalay, N.; Ott, S.; Brotman, R.M.; Ravel, J. An improved dual-indexing approach for multiplexed 16S rRNA gene sequencing on the Illumina MiSeq platform. Microbiome 2014, 2, 1-6. [CrossRef]

17. Igolkina, A.A.; Grekhov, G.A.; Pershina, E.V.; Samosorov, G.G.; Leunova, V.M.; Semenov, A.N.; Baturina, O.A.; Kabilov, M.R.; Andronov, E.E. Identifying components of mixed and contaminated soil samples by detecting specific signatures of control $16 \mathrm{~S}$ rRNA libraries. Ecol. Ind. 2018, 94, 446-453. [CrossRef]

18. Edgar, R.C. UPARSE: Highly accurate OTU sequences from microbial amplicon reads. Nat. Meth. 2013, 10, 996-998. [CrossRef]

19. Edgar, R.C. SINTAX, a Simple Non-Bayesian Taxonomy Classifier for 16S and ITS Sequences. bioRxiv 2016. [CrossRef]

20. Wang, Q.; Garrity, G.M.; Tiedje, J.M.; Cole, J.R. Nanve Bayesian Classifier for Rapid Assignment of rRNA Sequences into the New Bacterial Taxonomy. Appl. Environ. Microbiol. 2007, 73, 5261-5267. [CrossRef]

21. Hammer, O.; Harper, D.A.T.; Ryan, P.D. PAST: Paleontological Statistics Software Package for Education and Data Analysis. Palaeontol. Electron. 2001, 4, 9.

22. Wang, Q.; Hao, C.; Yao, W.; Zhu, D.; Lu, H.; Li, L.; Ma, B.; Sun, B.; Xue, D.; Zhang, W. Intestinal flora imbalance affects bile acid metabolism and is associated with gallstone formation. BMC Gastroenterol. 2020, 20, 59. [CrossRef]

23. Gutiérrez-Díaz, I.; Molinero, N.; Cabrera, A.; Rodríguez, J.I.; Margolles, A.; Delgado, S.; González, S. Diet: Cause or Consequence of the Microbial Profile of Cholelithiasis Disease? Nutrients 2018, 10, 1307. [CrossRef]

24. Vaiserman, A.; Romanenko, M.; Piven, L.; Moseiko, V.; Lushchak, O.; Kryzhanovska, N.; Guryanov, V.; Koliada, A. Differences in the gut Firmicutes to Bacteroidetes ratio across age groups in healthy Ukrainian population. BMC Microbiol. 2020, $20,221$. [CrossRef]

25. Rinninella, E.; Cintoni, M.; Raoul, P.; Lopetuso, L.R.; Scaldaferri, F.; Pulcini, G.; Miggiano, G.A.D.; Gasbarrini, A.; Mele, M.C. Food Components and Dietary Habits: Keys for a Healthy Gut Microbiota Composition. Nutrients 2019, 11, 2393. [CrossRef] [PubMed]

26. Vítek, L.; Carey, M.C. New pathophysiological concepts underlying pathogenesis of pigment gallstones. Clin. Res. Hepatol. Gastroenterol. 2012, 36, 122-129. [CrossRef]

27. Stender, S.; Frikke-Schmidt, R.; Nordestgaard, B.G.; Tybjærg-Hansen, A. Extreme Bilirubin Levels as a Causal Risk Factor for Symptomatic Gallstone Disease. JAMA Intern. Med. 2013, 173, 1222-1228. [CrossRef]

28. Di Ciaula, A.; Wang, D.Q.; Portincasa, P. An update on the pathogenesis of cholesterol gallstone disease. Curr. Opin. Gastroenterol. 2018, 34, 71-80. [CrossRef]

29. Gandy, K.; Zhang, J.; Nagarkatti, P.; Nagarkatti, M. The role of gut microbiota in shaping the relapse-remitting and chronicprogressive forms of multiple sclerosis in mouse models. Sci. Rep. 2019, 9, 6923. [CrossRef]

30. Kozhieva, M.; Naumova, N.; Alikina, T.; Boyko, A.; Vlassov, V.; Kabilov, M. Primary progressive multiple sclerosis in a Russian cohort: Relationship with gut bacterial diversity. BMC Microbiol. 2019, 19, 309. [CrossRef]

31. Jayachandran, M.; Chung, S.S.M.; Xu, B. A critical review of the relationship between dietary components, the gut microbe Akkermansia muciniphila, and human health. Crit. Rev. Food Sci. Nutr. 2019, 60, 1-12. [CrossRef]

32. Keren, N.; Konikoff, F.M.; Paitan, Y.; Gabay, G.; Reshef, L.; Naftali, T.; Gophna, U. Interactions between the intestinal microbiota and bile acids in gallstones patients. Environ. Microbiol. Rep. 2015, 7, 874-880. [CrossRef] [PubMed]

33. Ren, X.; Xu, J.; Zhang, Y.; Chen, G.; Zhang, Y.; Huang, Q.; Liu, Y. Bacterial Alterations in Post-Cholecystectomy Patients Are Associated with Colorectal Cancer. Front. Oncol. 2020, 10, 1418. [CrossRef] [PubMed]

34. Vacca, M.; Celano, G.; Calabrese, F.M.; Portincasa, P.; Gobbetti, M.; de Angelis, M. The Controversial Role of Human Gut Lachnospiraceae. Microorganisms 2020, 8, 573. [CrossRef] [PubMed]

35. Koh, A.; de Vadder, F.; Kovatcheva-Datchary, P.; Bäckhed, F. From Dietary Fiber to Host Physiology: Short-Chain Fatty Acids as Key Bacterial Metabolites. Cell 2016, 165, 1332-1345. [CrossRef]

36. La Rosa, S.L.; Leth, M.L.; Michalak, L.; Hansen, M.E.; Pudlo, N.A.; Glowacki, R.; Pereira, G.; Workman, C.T.; Arntzen, M.Ø.; Pope, P.B.; et al. The human gut Firmicute Roseburia intestinalis is a primary degrader of dietary $\beta$-mannans. Nat. Comm. 2019, 10, 905. [CrossRef]

37. Chow, J.; Tang, H.; Mazmanian, S.K. Pathobionts of the gastrointestinal microbiota and inflammatory disease. Curr. Opin. Immunol. 2011, 23, 473-480.

38. Chen, J.; Wright, K.; Davis, J.M.; Jeraldo, P.; Marietta, E.V.; Murray, J.; Nelson, H.; Matteson, E.L.; Taneja, V. An expansion of rare lineage intestinal microbes characterizes rheumatoid arthritis. Genome Med. 2016, 8, 43. [CrossRef]

39. Gomez-Arango, L.F.; Barrett, H.L.; Wilkinson, S.A.; Callaway, L.K.; McIntyre, H.D.; Morrison, M.; Dekker Nitert, M. Low dietary fiber intake increases Collinsella abundance in the gut microbiota of overweight and obese pregnant women. Gut Microbes 2018, 9, 189-201. [CrossRef]

40. Astbury, S.; Atallah, E.; Vijay, A.; Aithal, G.P.; Grove, J.I.; Valdes, A.M. Lower gut microbiome diversity and higher abundance of proinflammatory genus Collinsella are associated with biopsy-proven nonalcoholic steatohepatitis. Gut Microbes 2020, 11, 569-580. [CrossRef]

41. Heinken, A.; Ravcheev, D.A.; Baldini, F.; Heirendt, L.; Fleming, R.M.; Thiele, I. Systematic assessment of secondary bile acid metabolism in gut microbes reveals distinct metabolic capabilities in inflammatory bowel disease. Microbiome $2019,7,75$. [CrossRef] 
42. Corrêa, T.; Rogero, M.M.; Hassimotto, N.; Lajolo, F.M. The Two-Way Polyphenols-Microbiota Interactions and Their Effects on Obesity and Related Metabolic Diseases. Front. Nutr. 2019, 6, 188. [CrossRef]

43. Wang, W.; Wang, J.; Li, J.; Yan, P.; Jin, Y.; Zhang, R.; Yue, W.; Guo, Q.; Geng, J. Cholecystectomy Damages Aging-Associated Intestinal Microbiota Construction. Front. Microbiol. 2018, 9, 1402. [CrossRef]

44. Yoon, W.J.; Kim, H.N.; Park, E.; Ryu, S.; Chang, Y.; Shin, H.; Kim, H.L.; Yi, S.Y. The Impact of Cholecystectomy on the Gut Microbiota: A Case-Control Study. J. Clin. Med. 2019, 8, 79. [CrossRef]

45. Nahata, M.C.; Barson, W.J. Ceftriaxone: A third-generation cephalosporin. Drug Intell. Clin. Pharm. 1985, 19, 900-906.

46. Zhao, Z.; Wang, B.; Mu, L.; Wang, H.; Luo, J.; Yang, Y.; Yang, H.; Li, M.; Zhou, L.; Tao, C. Long-Term Exposure to Ceftriaxone Sodium Induces Alteration of Gut Microbiota Accompanied by Abnormal Behaviors in Mice. Front. Cell Infect. Microbiol. 2020, 10, 258. [CrossRef]

47. Giuffrè, M.; Campigotto, M.; Campisciano, G.; Comar, M.; Crocè, L.S. A story of liver and gut microbes: How does the intestinal flora affect liver disease? A review of the literature. Am. J. Physiol. Gastrointest. Liver Physiol. 2020, 318, G889-G906. [CrossRef] 